\section{Evaluation of Maximum and Minimum Signal Intensity and the Linear Relationship between Concentration and Signal Intensity in Saturation Recovery T1-weighted Images by use of a Turbo Fast Low-Angle Shot Sequence}

\author{
Nazarpoor M. ${ }^{1 * \odot(\odot) ~}$
}

\begin{abstract}
Background: The relationship between the concentration of contrast agents and signal intensity (SI) are affected by some image parameters, phase-encoding scheme, magnetic field strength, image sequences, and iron oxide nanoparticles used and GdDTPA as MRI contrast agents.
\end{abstract}

Objective: In this article, the effect of saturation times (TSs) on the maximum and minimum SI, and also the linear relationship between the concentration of the contrast agent and SI are evaluated. Additionally, we evaluated the concentration of contrast agent that results the minimum SI using a saturation recovery TurboFLASH sequence.

Material and Methods: In this experimental study, a phantom was designed to hold vials with different concentrations of Gd-DTPA $(0-19.77 \mathrm{mmol} / \mathrm{L})$. The mean SI was acquired from the nine central pixels of every vial at various TSs.

Results: This study shows that the maximum SI in an image is dependent on short TSs (up to $400 \mathrm{~ms}$ ) and independent of long TSs $(400-1000 \mathrm{~ms})$. The result also shows that the concentration at which a maximum linear relationship between concentration and $\mathrm{SI}$ is maintained that gave an $\mathrm{R}^{2}$ equal to 0.95 and 0.99 dependent on the TS. Moreover, the outcome demonstrates that as TS increases, the concentration of the contrast agent decreases. This causes SI to be minimized.

Conclusion: This study demonstrated that the TS is a key parameter for measuring the maximum and minimum SI and also TS plays the role in determining the maximum linear relationship between the MRI contrast agent concentration and SI in an in vivo perfusion study.

Citation: Nazarpoor M. Evaluation of Maximum and Minimum Signal Intensity and the Linear Relationship between Concentration and Signal Intensity in Saturation Recovery T1-weighted Images by use of a Turbo Fast Low-Angle Shot Sequence. J Biomed Phys Eng. 2020;10(4):449458. doi: $10.31661 /$ jbpe.v0i0.823.

\section{Keywords}

Magnetic Resonance Imaging; Saturation Recovery; T1-weighted; Signal Intensity; Saturation Time; Perfusion; Gadolinium DTPA

\section{Introduction}

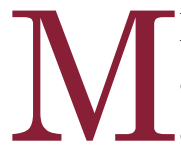
RI signal intensity (SI) alterations are related to the contrast agent (e.g., Gd-DTPA gadolinium-diethylenetriamine pentaacetic acid), or ultrasmall superparamagnetic iron oxide (USPIO) nanoparticles), strength of magnetic field, parameters of pulse sequence, phase-encoding scheme, rate and the volume of bolus injection
${ }^{1} \mathrm{PhD}$, Associated Professor of Medical Physics,

Department of Biomedi-

cal Engineering, Faculty

of Health, Tehran Medi-

cal Sciences, Islamic

Azad University, Tehran,

Iran

*Corresponding author: M. Nazarpoor

Associated Professor of Medical Physics, Department of Biomedical

Engineering, Faculty of Health, Tehran Medical

Sciences, Islamic Azad

University, Tehran, Iran

E-mail: mnazarpoor@

yahoo.co.uk

Received: 27 August 2017 Accepted: 10 October 2017 
of contrast agent, blood volume, the output of cardiac, and tissue topology [1-7]. It should be noted that the analysis of concentration-time can provide perfusion parameters (e.g., cerebral blood volume and flow, time to peak, and mean transit time). The concentration-time curve cannot be drawn directly in MRI. To do this, there should be a linear relationship between the concentration of contrast agent and SI $[8,9]$. The relation between the concentration and SI changes can be considered to be linear at low concentrations. To maximize the concentration [10], which leads to increase in the signal to noise ratio (SNR) in absolute perfusion measurements, the range of concentrations, where this linear relationship is maintained, should be known [8]. There isn't any fixed value for the relationship between concentration and SI regarding different publications [11-16].

In previous studies, we have investigated how the relation between the concentration of contrast agent and SI are affected by the image parameters like the repetition time (TR) [17], inversion time (TI) [18, 19], saturation time (TS) [18], echo time (TE) [20], flip angle [21], phase-encoding scheme [7], magnetic field strength [4], image sequences [5], and the use of Gd-DTPA [1] and iron oxide nanoparticles as contrast agents [2]. This work investigated the effect of saturation times (TSs) on the maximum and minimum SI, and also the relationship between SI and concentration using saturation recovery (SR) TurboFLASH (Turbo Fast Low-Angle Shot) (center out phase-encoding [7]) T1-weighted images. The linear relationship between concentration and SI was considered where the squared correlations $\left(\mathrm{R}^{2}\right)$ were equal to 0.95 and 0.99 . Another aim of this study was to evaluate the effect of changes in TS on the concentration of the contrast agent that results the minimum SI.

\section{Material and Methods}

In this experimental study, the following equation is used to measure the SI in T1- weighted inversion recovery (IR) sequences [6]:

$S(t)=S_{0}\left(1-\left(1-\cos \theta_{i n v}\right) \exp \frac{-T I}{T 1}+\exp \frac{-T R}{T 1}\right)(1)$

$\mathrm{S}(\mathrm{t})$ is the SI after injection of MRI contrast agent. $\mathrm{S}_{0}$ is the $\mathrm{SI}$ in the region of interest in the absence of contrast agent and $\theta_{\text {inv }}$ is flip angle of the inversion pulse. If $\theta_{\text {inv }}=90^{\circ}$, for the SR sequence Equation 1 is modified as follows:

$S(t)=S_{0}\left(1-\exp \frac{-T S}{T 1}+\exp \frac{-T R}{T 1}\right)$

In the above equation, TS is the time between the two pulses (saturation $90^{\circ}$ and $\alpha$ excitation). The relationship between $\mathrm{T} 1$ and concentration is described by the following formula [8]:

$\frac{1}{T 1}=\frac{C(t)}{K}+\frac{1}{T 1_{\text {Pre }}}$

Where $C(t)$ is the concentration of contrast agent at time $t$. $K$ is a constant which depends on the contrast media. $\mathrm{T} 1_{\mathrm{Pre}}$ is the longitudinal relaxation time at time $t$ before the application of contrast agent. Combining equation 2 and 3 leads to the following equation.

$S(t)=S_{0}\left(1-\exp \left(-T S\left(\frac{C(t)}{K}+\frac{1}{T 1_{\mathrm{Pre}}}\right)\right)+\exp \left(-T R\left(\frac{C(t)}{K}+\frac{1}{T 1_{\mathrm{pre}}}\right)\right)\right)(4)$

It should be noted that equations 1 and 4 at low concentrations are linear and in high concentrations non-linear.

At higher concentrations of contrast agents, equations 1 and 4 should be multiplied by a factor of $\exp \left(-\frac{T E}{T 2}\right)$ [22]. Where T2 and TE are the transverse relaxation time and echo time.

Figure 1 illustrates a schematic diagram of the SR gradient echo TurboFLASH sequence.

In SR TurboFLASH, the $90^{\circ}$ pulse is just a saturation pulse (the excitation pulses are $\alpha$ pulses). Therefore, SR TurboFLASH is more analogous to IR TurboFLASH, except that the magnetization preparation is a $90^{\circ}$ pulse rather 


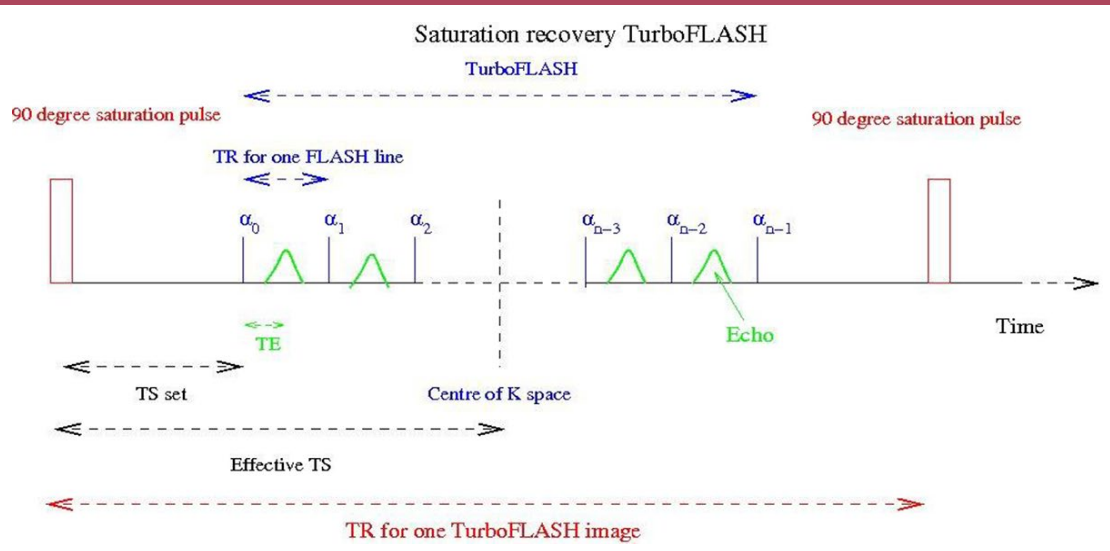

Figure 1: Saturation recovery (SR) gradient echo TurboFLASH sequence with $n$ a pulses for one image without showing the imaging gradients. Where the $n / 2$ line traverses the center of $K$ space. "Saturation times (TS) set" is the time between the two pulses $\left(90^{\circ}\right.$ saturation and the first $\alpha$ excitation).

than a $180^{\circ}$ pulse [8]. Above all, the concept of a TS is appropriate for SR TurboFLASH, in the same manner as TI is applied to the IR TurboFLASH [7]. For SR spin echo, TS $\equiv$ TR, however, the two parameters are different for SR TurboFLASH.

\section{Phantom}

To assess the effect of TS on the SI at different concentrations, a cubic Perspex phantom with the dimensions of 20,18 , and $20 \mathrm{~cm}$ was designed to hold vials (glass tube, $15 \mathrm{~mm}$ diameter, contain 27 pixels) containing constant or different concentrations of the contrast agent [7]. One important factor in non-uniformity of an image is radio frequency (RF) coil inhomogeneity in the MR scanner [23]. The vials of constant concentration $(1.20 \mathrm{mmol} / \mathrm{L})$ were used to measure coil non-uniformity. The vials of different concentrations were used to evaluate the relation between concentration and SI. The vials are prepared by different concentrations of Gd-DTPA $(0-19.77 \mathrm{mmol} / \mathrm{L}$ with values of $0.00,0.30,0.45,0.60,0.75$, $0.90,1.20,1.50,1.80,2.10,2.39,2.69,2.99$, $3.28,3.58,3.98,4.96,5.95,7.93,9.90,13.85$, and $19.77 \mathrm{mmol} / \mathrm{L})$. Two different images were taken the vials with different and constant concentrations. Then the non-uniformity of coil was measured by SI of each vial with constant concentration. To find a correction factor, the obtained SI in the region of interest was normalized.

To calculate the corrected SI for each vial with different concentrations, the SI was multiplied by its correction factor. The positions of the vials in the phantom with the constant concentration and the vials with different concentrations were the same.

\section{Image acquisition}

The phantom was placed in the clinical head and neck coil in MRI scanner (1.5 T, Vision). For measuring SI, TurboFLASH T1-weighted images were repeated 10 times with the vials of different and constant concentration.

According to the imaging parameters, the matrix size $=128 \times 128, \mathrm{TR}=10 \mathrm{~s}, \mathrm{TE}=4$ $\mathrm{ms}$, TS varied between 50 and $1000 \mathrm{~ms}$ (50, $100,150,200,300,400,500,600,800$, and $1000 \mathrm{~ms}$ ), pixel size $=2 \times 2 \mathrm{~mm}$, flip angle $=$ $10^{\circ}$, and slice thickness $=10 \mathrm{~mm}$.

The image data were transmitted from the MR scanner to a personal computer. Interactive Data Language (IDL, Research Systems, Inc. http://www.rsinc.com) was used for image processing.

Programs of IDL were written to automatically find the following:

1- The mean image of 10 acquisitions, for 
improving the SNR.

2- The mean SI and standard deviation of the nine out of 27 central pixels to avoid partial volume effects.

3- The correction factors for the non-uniformity of coil using the vials with constant concentration. The corrected SI is calculated by multiplying these factors by SI of the vials with different concentrations.

4- The best-fit curve of concentration versus SI using equation 4.

5- The concentration of contrast agent is marked that leads to maximum and minimum SI at different TSs.

6- The maximum concentration where the $\mathrm{R}^{2}$ of best-fit curve in the plot of corrected SI against concentration was equal to 0.95 or 0.99 . $\mathrm{R}^{2}$ gives the strength of the linear relationship between concentration and SI. When $\mathrm{R}^{2}=0.95$, it shows that $95 \%$ of the variation in SI is caused by the variation of concentration [24].

Personal computer or a UNIX workstation could be used for programs running.

\section{Results}

Figure 2 shows a typical result for the corrected SI versus concentration of contrast agent at TS $=600 \mathrm{~ms}$ for a T1-weighted SR TurboFLASH sequence. The dashed lines demonstrate the best-fit curves for the data using equation 4.

The mean SI was measured from the nine central pixels of each vial. The non-uniformity coil correction factors were applied to the mean SI to find the corrected SI. The correction factors were $0.96,0.96,1.00,1.00,0.94,0.98$, $1.00,1.00,0.97,0.94,0.93,1.03,0.99,0.95$, $1.00,1.06,0.98,1.01,0.99,1.03,1.06$, and 1.01 for the vials with concentration of 0.00 , $0.30,0.45,0.60,0.75,0.90,1.20,1.50,1.80$, 2.10, 2.39, 2.69, 2.99, 3.28, 3.58, 3.98, 4.96, 5.95, 7.93, 9.90, 13.85, and $19.77 \mathrm{mmol} / \mathrm{L}$, respectively. As seen in the figure, the maximum SI (380.39 \pm 19.09 , mean \pm standard deviation) was at $5.95 \mathrm{mmol} / \mathrm{L}$. When $\mathrm{R}^{2} \mathrm{~s}$ are

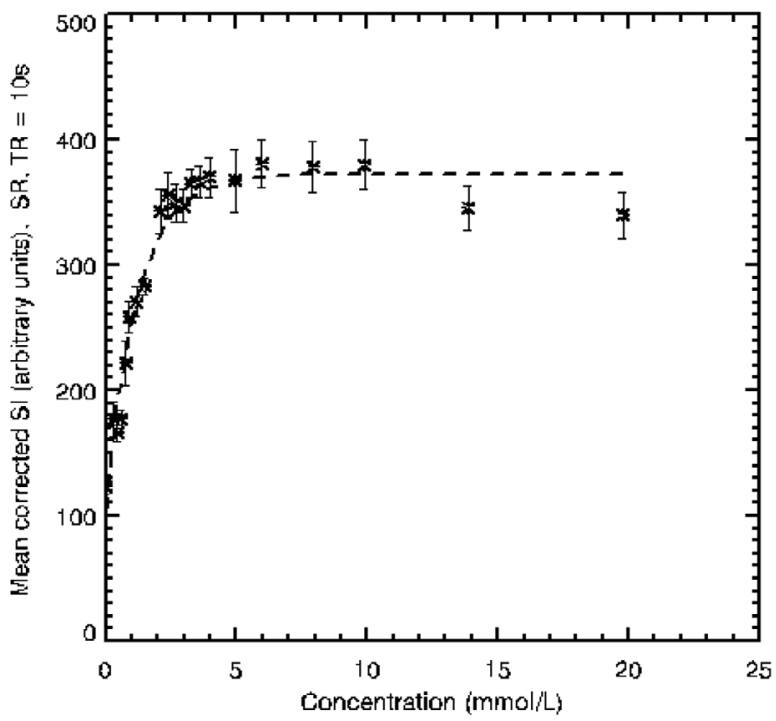

Figure 2: Mean corrected SI versus the contrast agent concentration. The dashed lines demonstrate the best-fit curves for the data. The maximum concentrations, at which a linear relationship between corrected $\mathrm{SI}$ and concentrations was maintained, in $\mathrm{R}^{2}=0.95$ and 0.99 were 2.15 and $0.96 \mathrm{mmol} / \mathrm{L}$, respectively, at TS $=600 \mathrm{~ms}$, which were calculated by best-fit curve. The standard deviations of SI from nine innermost pixels of each vial are shown by error bars.

equal to 0.95 and 0.99 , the linear relationship between corrected SI and concentrations were measured as 2.15 and $0.96 \mathrm{mmol} / \mathrm{L}$, respectively. The best-fit curve was applied to the data for measuring $\mathrm{R}^{2}$.

Figure 3 shows the other typical results of corrected SI versus concentration of contrast agent at $\mathrm{TS}=200 \mathrm{~ms}$. At concentration of $9.90 \mathrm{mmol} / \mathrm{L}$, the SI was maximum $(363.52$ $\pm 18.45)$. The maximum linear relationship between concentrations and corrected SI happened at 4.78 and $2.08 \mathrm{mmol} / \mathrm{L}$ for an $\mathrm{R}^{2}$ of 0.95 and 0.99 , respectively, which were calculated using the best-fit curve based on equation 4.

The minimum SI was found at contrast agent concentrations of 0.6 and $0.45 \mathrm{mmol} / \mathrm{L}$ at a TS of $200 \mathrm{~ms}(63.84 \pm 5.47)$ and $600 \mathrm{~ms}(165.91$ \pm 7.09 ), respectively (see Figures 2 and 3 ). 


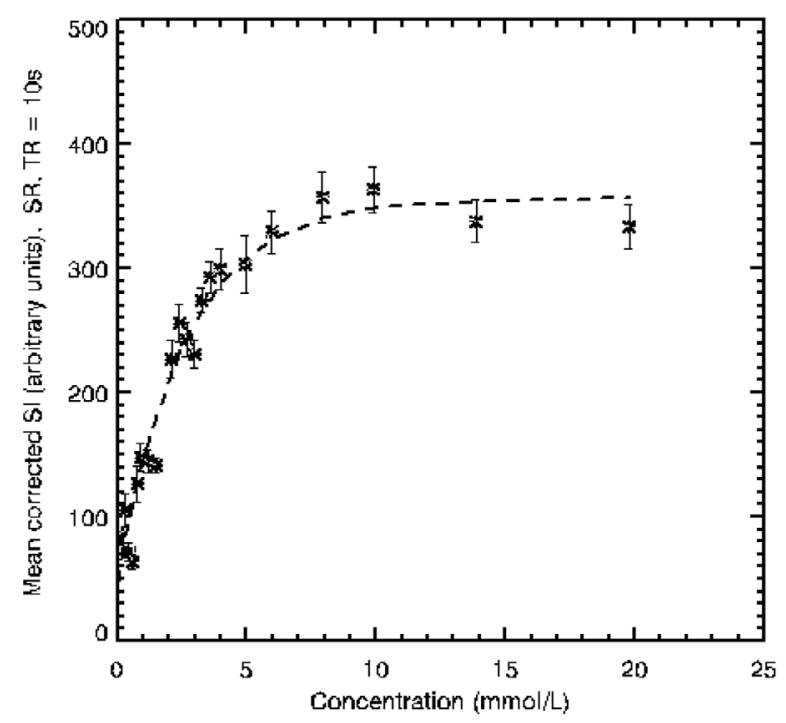

Figure 3: Mean corrected SI versus concentration of the contrast agent at TS $=200$ ms. The best-fit curve for the data based on equation 4 is shown by dashed lines. The error bars illustrate the standard deviation of SI from nine innermost pixels of each vial.

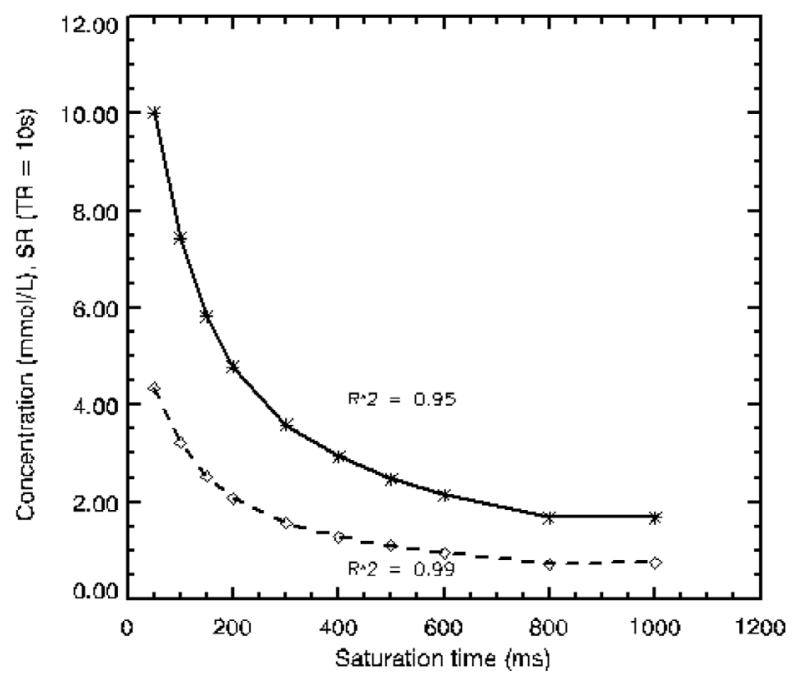

Figure 4: Maximum concentration that yields $R^{2}=0.95$ and 0.99 versus TS. For each TS, the $R^{2}$ was calculated from the best-fit curve.
Figure 4 shows the maximum concentration values which resulted in 0.95 and 0.99 for $\mathrm{R}^{2}$ versus 10 different TS values. The detailed values of $\mathrm{R}^{2}$ were obtained similar to Figures 2 and 3 for each TS. Regarding the maximum linear relationship between concentration of contrast agent and SI, Figure 4 shows a concentration of $1.68 \mathrm{mmol} / \mathrm{L}$ and $10.02 \mathrm{mmol} / \mathrm{L}$ for a long TS (1000 ms) and short TS (50 ms), respectively, where $\mathrm{R}^{2}=0.95$. When $\mathrm{R}^{2}=$ 0.99 , these values decreased to $0.75 \mathrm{mmol} / \mathrm{L}$ and $4.35 \mathrm{mmol} / \mathrm{L}$ for the same TSs.

Figure 5 shows TS versus maximum SI. The maximum SI was found at a lower concentration of contrast agent, when there is a dominant T1-shortening effect. The figure indicates that the maximum SI increased up to a TS of $400 \mathrm{~ms}$. In addition, the maximum SI was nearly the same for long TSs (400-1000 ms). This means that the maximum SI of an image is independent of TS at long TSs.

Figure 6 displays the TS versus minimum SI. The figure indicates that at a TS between 50 and $800 \mathrm{~ms}$, the minimum SI increased. In

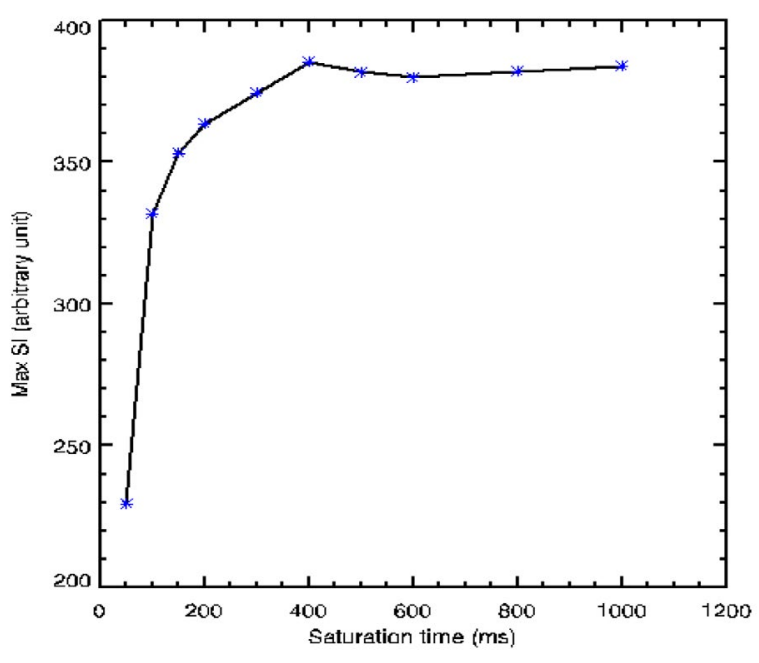

Figure 5: TS versus maximum SI. The figure indicates that the maximum $\mathrm{SI}$ increased up to a TS of $400 \mathrm{~ms}$. In addition, the maximum SI was nearly the same at long TSs (400-1000 $\mathrm{ms})$. 


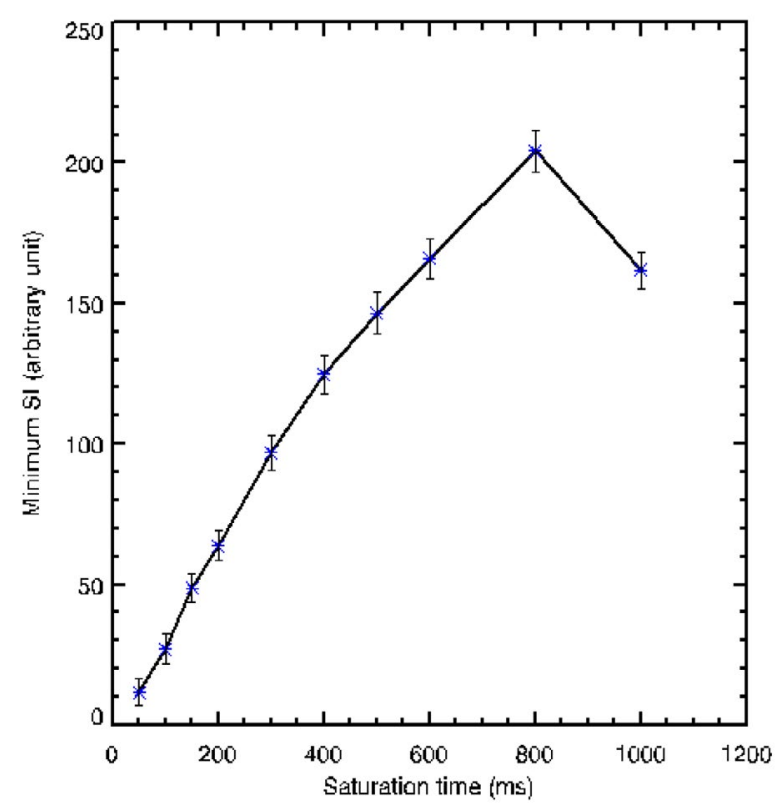

Figure 6: TS versus minimum SI. The figure indicates that at TS between 50 and $900 \mathrm{~ms}$, the minimum $\mathrm{SI}$ increased with increasing TS. In addition, at a long TS (1000 ms), the minimum SI decreased. It is necessary to mention that the SI started at a high value and gradually decreased and, subsequently, increased again. The minimum $\mathrm{SI}$ is dependent on the concentration and TS. The standard deviation of the nine central pixels of each vial was shown by error bars.

addition, at a longer TS (1000 ms), the minimum SI decreases.

Figure 7 demonstrates TS versus the concentration that results to the minimum SI. Contrast agent concentration and TS affected the minimum SI.

\section{Discussion}

$\mathrm{T} 1$ and $\mathrm{T} 2$ relaxation times will decrease after the injection of contrast agent. Both the T1- and T2-shortening effect can influence SI on MRI. The T1 shortening effect is predominant at low contrast agent concentrations, as modeled by equation 4 , and results to an increase in the SI. The T2 shortening effect is prominent at high concentrations and leads to a decline in the SI [7]. Both T1 and T2 can be influenced at high concentrations, as the SI response displays a non-linear relationship with an unsteady plateau (see Figures 2 and 3).

The concentration of the contrast agent for perfusion study can be calculated indirectly from the SI on MRI. The concentration of the contrast agent does not necessarily correlate linearly with SI on MRI. Therefore, in order to calculate the concentration from SI, the maximum concentration, where a linear relationship is maintained, should be measured [7]. T1-weighted images need approximately $1 / 10$ th of the volume of contrast agent that is normally used for a T2*-weighted acquisition in a perfusion study $[8,25]$. Small volumes of contrast agent leads to a reduction in the SNR. By the injection of more contrast agents, SNR ratio should be improved. TS is one of the important parameters for SI measurement be-

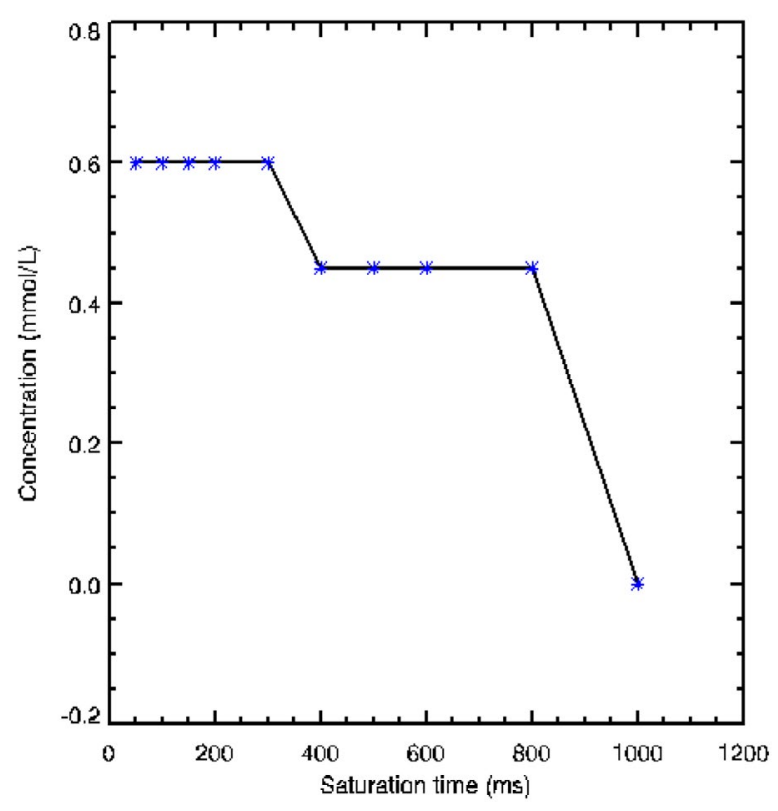

Figure 7: TS versus concentration that leads to the minimum SI. The minimum SI appeared at a concentration of 0.6 and 0.4 $\mathrm{mmol} / \mathrm{L}$ for TSs between 50 and $300 \mathrm{~ms}$, and TSs between 400 and 800 ms, respectively. 
cause it can have an effect on the maximum concentration where there is a linear relationship between SI and the concentration of the contrast agent. Canet et al. presented the correlation between the concentration of GdDOTA (Gadoterate, $0.5 \mathrm{~mol} / \mathrm{mL}$ ) and SI using T1-weighted IR TurboFLASH [11]. They investigated a broad range of concentrations (0-5.0 mmol/L, Gd-DOTA diluted in saline, which has approximately the same relaxation time as Gd-DTPA [16]) in vitro. They used a 1.5 T MR scanner with an inversion time of $300 \mathrm{~ms}$ to obtain the MR image. They reported a linear relationship between SI which is less than $0.8 \mathrm{mmol} / \mathrm{L}$ of concentration and a nonlinear one at higher concentrations. They also indicated that the maximum SI was apparent at the concentration of $4 \mathrm{mmol} / \mathrm{L}$. The present study found that if TS increases, the maximum concentration will decrease that demonstrates a linear relationship with the SI.

Our results using Gd-DTPA as the contrast agent in an SR sequence were approximately $3.60 \mathrm{mmol} / \mathrm{L}$ and $1.57 \mathrm{mmol} / \mathrm{L}$ for $\mathrm{R}^{2}=0.95$ and 0.99 , respectively, with $\mathrm{TR}=10 \mathrm{~s}$ and $\mathrm{TS}$ $=300 \mathrm{~ms}$ (see Figure. 4 ). In addition, the maximum SI was found at a concentration of 9.9 $\mathrm{mmol} / \mathrm{L}$. The difference between this studies in comparison with Canet et al.'s report is using a different image sequence and a different value of $R^{2}[11]$. Neither of these values $\left(R^{2}=\right.$ 0.95 and 0.99 ) were given by Canet. Takeda et al. investigated the relationship between the SI and concentration of contrast agent (GdDTPA) by the use of T1-weighted images [12]. They presented that the linear relationship between concentration of Gd-DTPA and SI was up to a concentration of $2.0 \mathrm{mmol} / \mathrm{L}$ ( TS $=100 \mathrm{~ms}$, flip angle $=60^{\circ}$ or $90^{\circ}$, magnetic field strength $=0.1 \mathrm{~T}, \mathrm{R}^{2}=0.76$ ) using SR (gradient echo) sequences. The linearity extended up to $2.0\left(\mathrm{R}^{2}=0.94, \mathrm{TS}=500 \mathrm{~ms}\right)$ for spin-echo sequence and up to $3.0 \mathrm{mmol} / \mathrm{L}$ (TS $=34 \mathrm{~ms}$, flip angle $=45^{\circ}$, magnetic field strength $=0.1 \mathrm{~T}, \mathrm{R}^{2}=0.81$ ) for gradient-echo sequences. One of Takeda's report is in line with the present study; when TS $=500 \mathrm{~ms}$, there was a linear relationship up to a concentration of $2.49 \mathrm{mmol} / \mathrm{L}$ at $\mathrm{R}^{2}=0.95$ or up to $1.10 \mathrm{mmol} / \mathrm{L}$ at $\mathrm{R}^{2}=0.99$ (see Figure 4). In addition, our results illustrated that the linearity was dependent on the $\mathrm{R}^{2}$ value chosen, concentration, and the TS of the SR TurboFLASH sequence. The difference between the findings of current study and those of Takeda et al. likely is due to differences in image parameters (flip angle and magnetic field strength) [4], and perhaps the method of calculating the maximum concentration where there is a linear relation. A linear relationship between changes in concentrations up to $1.0 \mathrm{mmol} / \mathrm{L}$ and SI using an IR TurboFLASH sequence (linear phase-encoding, effective TI $=720 \mathrm{~ms}$ ) was reported by Fritz-Hansen et al. [13]. As the image sequence (SR TurboFLASH) and phase acquisition (center-out phase-encoding) of current study were different with those of Fritz-Hansen's study. Therefore, it is impossible to compare them. Our previous investigation demonstrated that the phase-encoding scheme and image sequence could have an effect on the relation between MRI contrast agent concentration and SI [5, 7]. Mørkenborg et al. found that there was a linear relationship between Gd-DTPA concentration and SI in the range of $0-5.0\left(\mathrm{R}^{2} \geq 0.95\right)$ and $0-3.0 \mathrm{mmol} / \mathrm{L}$ $\left(\mathrm{R}^{2} \geq 0.92\right)$ in 1.5 and $7 \mathrm{~T}$ systems, respectively, when using a spin echo sequence [14]. In addition, Dean et al., and Fritz-Hansen et al., and Unger et al. stated that this linearity can be found up to $1 \mathrm{mmol} / \mathrm{L}$ using an IR sequence. Since our image sequence was differed from those of Mørkenborg's, Dean's, Fritz-Hansen's, and Unger's, it is impossible to compare their findings with our results $[13,16]$.

The effect of TS change on the linear relationship between contrast agent and SI, with concentrations maintaining a threshold of $\mathrm{R}^{2}=0.95$ and 0.99 on SR TurboFLASH T1weighted images on MRI (TR $=2 \mathrm{~s}$, flip angle $=15^{\circ}$, and TS was varied between 300 and $800 \mathrm{~ms}$ ) was reported in our previous inves- 
tigation [18]. The results illustrated that the linearity was present up to 3.37 for $\mathrm{TS}=300$ $\mathrm{ms}$ and $1.46 \mathrm{mmol} / \mathrm{L}$ for $\mathrm{TS}=800 \mathrm{~ms}\left(\mathrm{R}^{2}=\right.$ 0.95). Furthermore, the reductions in values were 1.54 and $0.66 \mathrm{mmol} / \mathrm{L}$ when $\mathrm{R}^{2}=0.99$. This work studied the effect of TSs (50-1000 $\mathrm{ms}$ ) on the maximum and minimum SI, and the maximum concentration where there was a linear relationship between concentration and SI $\left(\mathrm{R}^{2}=0.99\right.$ and 0.95$)$ using SR TurboFLASH T1-weighted images at TR $=10 \mathrm{~ms}$ and flip angle of $10^{\circ}$. The present results indicate that there is a negative correlation between TS and maximum concentration where the relation is linear. The results also show that at a long TS (1000 ms), there is a linear relationship between concentration up to a concentration of $1.68 \mathrm{mmol} / \mathrm{L}$ and SI when $\mathrm{R}^{2}=0.95$ or up to $0.75 \mathrm{mmol} / \mathrm{L}$ when $\mathrm{R}^{2}=$ 0.99 . These concentrations may rise at short TS periods. Moreover, the results show that the maximum concentrations are 3.4 and 1.68 $\mathrm{mmol} / \mathrm{L}$ when there is a linear relationship $\left(\mathrm{R}^{2}\right.$ $=0.95$ ) for $\mathrm{TS}=300$ and $800 \mathrm{~ms}$ (similar to our previous study [18]), respectively. These concentrations were 1.68 and $0.75 \mathrm{mmol} / \mathrm{L}$ for the two TSs, respectively, when $\mathrm{R}^{2}=0.99$ (see Figure 4). The slight differences in the results in the present and previous study are due to the TR and flip angle differences [17]. In addition, our previous study did not measure the concentration and TSs which led to the maximum and minimum SI in details, thus it was fully compared with the results of this study (see Figures 5-7).

Figure 5 of this study suggests that an increase in TS (up to $400 \mathrm{~ms}$ ) is associated with an increase in the maximum SI. At all long TSs (400-1000 ms), the maximum SI was nearly the same. This means that the maximum SI on an image is independent of TS for long TSs.

Figures 6 indicates that TS can have an effect on the minimum SI. The result indicates that an increment in TS leads to an increase in the minimum SI up to TS $=800 \mathrm{~ms}$ and a decrease for TS $=1000 \mathrm{~ms}$.
Figure 7 indicates that the minimum SI was apparent at a concentration of $0.6 \mathrm{mmol} / \mathrm{L}$ for TSs between 50 and $300 \mathrm{~ms}$, and $0.45 \mathrm{mmol} / \mathrm{L}$ for TSs between 400 and $800 \mathrm{~ms}$. The figure shows that the concentration resulting in the minimum SI decreased at TS $=1000 \mathrm{~ms}$. Our previous study investigated the effect of TI values on the contrast agent concentration that causes minimum SI using an IR fast-gradient echo 3-dimensional T1-weighted image [26]. This study showed that for the lower $\mathrm{T}$, the $\mathrm{T} 1$ recovery curve did not cut off the x-axis. Thus, the minimum SI will be seen on the contrast agent concentration versus SI curves. Moreover, the findings suggested that the contrast agent concentration resulting a minimum SI (null point) is related to TI. At higher TI, the minimum SI will be expended. Despite the fact that IR sequences start with a $180^{\circ}$ inversion pulse the $\mathrm{T} 1$ recovery curve crossed the $\mathrm{X}$-axis (at lower TIs), the SR TurboFLASH started with $90^{\circ}$ saturation pulse and the longitudinal magnetization vector will be tip down into the $\mathrm{x}-\mathrm{y}$ plane. After a delay time (TS), $\mathrm{n}$ $\alpha$-pulses followed, therefore the longitudinal magnetization vector ( $\mathrm{T} 1$ recovery curve) did not cut off the x-axis. Consequently, the SI should increase with increased concentrations of the contrast agent in SR sequences, without consideration for the T2 effect at high concentrations that results a decrease in SI. Therefore, the minimum SI should not appear on SI versus contrast agent concentration curves (see Figures 2 and 3). One reason for the minimum SI at lower TSs and concentration could be due to noisy data. Since the standard deviations of the nine innermost pixels were not high at lower TSs in this study (see the error bar on Figure. 6), this is not the case. It seems that the phenomenon of a minimum SI on SR sequences at lower concentrations of contrast agent occurs for an unknown reason. Since the image parameters and image sequences are different from our previous study, it is impossible to compare the two results [26]. To our knowledge, no study has examined that effect 
to compare with our results using SR TurboFLASH T1-weighted images.

In summary, the linear correlation between the concentration of contrast agent (e.g. GdDTPA, Magnevist) and SI should be known in a perfusion measurement $[8,25,27]$. The $\mathrm{R}^{2}$ values for the short TS are higher than those for the long TS; therefore, to increase the SNR in clinical study, a short TS should be chosen. To calculate the optimal injection dose for specific contrast agent concentration in clinical studies, refer to our previous papers [5, 28].

\section{Conclusion}

This study found that the maximum SI of an image is dependent on short TSs (up to $400 \mathrm{~ms}$ ) and independent of long TSs (400-1000 ms) on T1-weighted SR TurboFLASH sequence. Our finding also shows that the maximum concentration at which a linear relationship maintained is related to the TS. An increment in the TS leads to a decrease in the range of concentrations with a linear relationship maintained. Therefore, the SNR may be improved by using a high volume of contrast agent with a low TS value for SR sequences. The results also show that the TS can have an effect on the contrast agent concentration, which results to the minimum SI. The concentration of the contrast agent, that would reduce the signal strength, can be avoided by the right choice of saturation time in clinical studies.

In conclusion, this study indicates that the TS is a key parameter for determining the maximum and minimum SI in a region of interest and also TS plays the role in determining the maximum linear relationship between the MRI contrast agent concentration and SI in an in vivo perfusion study.

\section{Acknowledgment}

For MRI data acquisition, the author gratefully acknowledges from Dr. Paul Morgan.

\section{Conflict of Interest}

None
References

1. Calamante F, Vonken EJ, Van Osch MJ. Contrast agent concentration measurements affecting quantification of bolus-tracking perfusion MRI. Magn Reson Med. 2007;58:544-53. doi: 10.1002/ mrm.21362. PubMed PMID: 17763347.

2. Gharehaghaji N, Nazarpoor M, Saharkhiz H. Effect of iron oxide nanoparticles coating type on the relationship between nanoparticles concentration and signal intensity in inversion recovery T1-weighted MRI. Med J Islam Repub Iran. 2015;29:211-20. doi: 10.5812/iranjradiol.22887.

3. Rohrer M, Bauer H, Mintorovitch J, Requardt M, Weinmann HJ. Comparison of magnetic properties of MRI contrast media solutions at different magnetic field strengths. Invest Radiol. 2005;40:71524. doi: 10.1097/01.rli.0000184756.66360.d3. PubMed PMID: 16230904.

4. Nazarpoor M, Mayabi Z, Shfaie A, Pesianian E, Aghaverdizadeh D. Maximum relationship between signal intensity and concentration of contrast agent in $0.3 \mathrm{~T}$ and $1.5 \mathrm{~T}$ using T1-weighted spin echo sequence. Medical Journal of Tabriz University of Medical Sciences \& Health services. 2011;32:72-6.

5. Nazarpoor M, Poureisa M, Daghighi MH. Comparison of maximum signal intensity of contrast agent on t1-weighted images using spin echo, fast spin echo and inversion recovery sequences. Iran J Radiol. 2012;10:27-32. doi: 10.5812/iranjradiol.5452. PubMed PMID: 23599710. PubMed PMCID: PMC3618902.

6. Bernstein MA, King KF, Zhou XJ. Handbook of MRI pulse sequences. London: Elsevier; 2004.

7. Nazarpoor M. Effect of phase-encoding scheme on the relationship between contrast agent concentration and signal intensity on inversion recovery Turbo Fast Low-Angle Shot T1-weighted images. Radiological physics and technology. 2014;7:26270. doi: 10.1007/s12194-014-0260-7.

8. Nazarpoor M. Organ Blood Flow Measurement with $\mathrm{T} 1$ and $\mathrm{T} 2^{*}$-Weighted MRI Techniques. Saarbrücken: Lap Lambert Academic Publ; 2012.

9. Lüdemann L, Warmuth $C$, Plotkin M, Förschler A, Gutberlet M, Wust $P$, et al. Brain tumor perfusion: Comparison of dynamic contrast enhanced magnetic resonance imaging using $\mathrm{T} 1$, $\mathrm{T} 2$, and $\mathrm{T} 2{ }^{*}$ contrast, pulsed arterial spin labeling, and $\mathrm{H} 2150$ positron emission tomography. Eur J Radiol. 2009;70:465-74. doi: 10.1016/j. ejrad.2008.02.012.

10. Nazarpoor M. Effect of concentration of contrast 
agent on the inflow effect for measuring absolute perfusion by use of inversion recovery $T$ 1-weighted TurboFLASH images. Radiological physics and technology. 2012;5:86-91.

11. Canet E, Douek P, Janier M, Bendid K, Amaya $J$, Millet $P$, et al. Influence of bolus volume and dose of gadolinium chelate for first-pass myocardial perfusion MR imaging studies. J Magn Reson Imaging. 1995;5:411-5. doi: 10.1002/ jmri.1880050407. PubMed PMID: 7549202.

12. Takeda M, Katayama $Y$, Tsutsui T, Komeyama $T$, Mizusawa T. Does gadolinium-diethylene triamine pentaacetic acid enhanced MRI of kidney represent tissue concentration of contrast media in the kidney? in vivo and in vitro study. Magn Reson Imaging. 1994;12:421-7. doi: 10.1016/0730$725 \times(94) 92535-6$.

13. Fritz-Hansen T, Rostrup E, Ring PB, Larsson HB. Quantification of gadolinium-DTPA concentrations for different inversion times using an IRturbo flash pulse sequence: a study on optimizing multislice perfusion imaging. Magn Reson Imaging. 1998;16:893-9. doi: 10.1016/s0730725x(98)00103-9. PubMed PMID: 9814771.

14. Morkenborg J, Pedersen M, Jensen FT, StodkildeJorgensen H, Djurhuus JC, Frokiaer J. Quantitative assessment of Gd-DTPA contrast agent from signal enhancement: an in-vitro study. Magn Reson Imaging. 2003;21:637-43. doi: 10.1016/s0730725x(03)00096-1. PubMed PMID: 12915195.

15. Dean BL, Lee C, Kirsch JE, Runge VM, Dempsey RM, Pettigrew LC. Cerebral hemodynamics and cerebral blood volume: MR assessment using gadolinium contrast agents and T1-weighted Turbo-FLASH imaging. American journal of neuroradiology. 1992;13:39-48.

16. Unger EC, Ugurbil K, Latchaw RE. Contrast agents for cerebral perfusion MR imaging. J Magn Reson Imaging. 1994;4:235-42. doi: 10.1002/ jmri.1880040303. PubMed PMID: 8061420.

17. Nazarpoor M. The effect of repetition time on the maximum linear relationship between contrast agent concentration and signal intensity on T1-weighted image using inversion recovery (IR) sequence. Iranian Journal of Radiology. 2009;6(4):247-52.

18. Nazarpoor M. Effects of inversion and saturation times on relationships between contrast agent concentrations and signal intensities of $T$ 1-weighted magnetic resonance images. Radiological physics and technology. 2010;3:120-6. doi: 10.1007/s12194-010-0087-9.
19. Nazarpoor M, Gharehaghaji N, Saharkhiz H. Effect of Inversion Time on the Linear Correlation between Polyethylene Glycol Coated Iron Oxide Nanoparticles Concentration and Signal Intensity in Inversion Recovery MRI Pulse Sequence. Journal of Mazandaran University of Medical Sciences. 2015;24:88-97.

20. Nazarpoor M, Poureisa M, Daghighi MH. Effect of echo time on the maximum relationship between contrast agent concentration and signal intensity using FLAIR sequence. Iranian Journal of Medical Physics. 2013;10:59-67.

21. Gharehaghaji N, Nazarpoor M, Saharkhiz H. Effect of Flip Angle on the Correlation Between Signal Intensity and Different Concentrations of Iron Oxide Nanoparticles Using T1-Weighted Turbo-FLASH Inversion Recovery Sequence. Iran J Radiol. 2015;12:e22887. doi: 10.5812/iranjradiol.22887. PubMed PMID: 25901260. PubMed PMCID: PMC4389174.

22. McRobbie DW, Moore EA, Graves MJ, Prince MR. MRI: Prom Picture to Proton. UK, Cambridge: Cambridge University Press; 2006.

23. Nazarpoor M. Non-uniformity of Clinical Head, Head and Neck, and Body Coils in Magnetic Resonance Imaging (MRI). Iranian Journal of Medical Physics. 2014;11:322-7.

24. Kleinbaum D, Kupper L, Muller K, Nizam A. Poisson regression analysis. Applied regression analysis and other multivariable methods. 1998;3:687709.

25. Nazarpoor M, Morgan P. Inflow effect correction in perfusion measurement of normal subjects with T1-weighted images using inversion recovery sequences. Iranian Journal of Radiology. 2010;7(3):145-51.

26. Nazarpoor $M$. The effect of inversion times on the minimum signal intensity of the contrast agent concentration using inversion recovery t1-weighted fast imaging sequence. Med J Islam Repub Iran. 2014;28:128.

27. Nazarpoor M. Evaluation of flow measurement from the first pass bolus $T 1$ weighted images using inversion recovery sequence. The British journal of radiology. 2011;84:342-9. doi: 10.1259/ bjr/18588668.

28. Nazarpoor M, Poureisa M, Daghighi M. Investigations of optimal dose of contrast agent concentration from routine dose using spin echo and inversion recovery T1-weighted sequences in MRI. Medical Journal of Tabriz University of Medical Sciences \& Health services. 2013;34:74-8. 